\title{
Application of the state-dependent riccati equation and kalman filter techniques to the design of a satellite control system
}

\author{
L.C.G. Souza* and R.G. Gonzáles \\ National Institute for Space Research- INPE, Brazilian Aeronautical Company-Embraer, São Jose dos Campos, SP, \\ Brasil
}

\begin{abstract}
Design of Satellite Attitude Control System (ACS) that involves plant uncertainties and large angle manoeuvres following a stringent pointing control, may require new non-linear control techniques in order to have adequate stability, good performance and robustness. In that context, experimental validation of new non-linear control techniques through prototypes is the way to increase confidence in the controller designed. The Space Mechanics and Control Division (DMC) of INPE is constructing a 3-D simulator to supply the conditions for implementing and testing satellite ACS hardware and software. The 3-D simulator can accommodate various satellites components; like sensors, actuators, computers and its respective interface and electronic. Depending on the manoeuvre the 3-D simulator plant can be highly non-linear and if the simulator inertia parameters are not well determined the plant also can present some kind of uncertainty. As a result, controller designed by linear control technique can have its performance and robustness degraded, therefore controllers designed by new non-linear approach must be considered. This paper presents the application of the State-Dependent Riccati Equation (SDRE) method in conjunction with Kalman filter technique to design a controller for the DMC 3-D satellite simulator. The SDRE can be considered as the non-linear counterpart of Linear Quadratic Regulator (LQR) control technique. Initially, a simple comparison between the LQR and SDRE controller is performed. After that, practical applications are presented to address problems like presence of noise in process and measurements and incomplete state information. Kalman filter is considered as state observer to address these issues. The effects of the plant non-linearities and noises (uncertainties) are considered in the performance and robustness of the controller designed by the SDRE and Kalman filter. The 3-D simulator simulink-based model has been developed to perform the simulations examples to investigate the SDRE controller performance using the states estimated by the Kalman filter. Simulations have demonstrated the validity of the proposed approach, once the SDRE controller has presented good stability margin, great performance and robustness.
\end{abstract}

Keywords: Satellite simulator, SDRE methodology, robust control

\section{Introduction}

There are several methodologies to investigate the satellite Attitude Control System (ACS) performance and robustness, when the investigation objectives are to validate hardware in the loop equipments experimental procedure can be more appropriate, some applications can be found in [1-3]. The use of experimental platforms has the important advantage of allowing the satellite dynamics representation in laboratory, from which is possible to accomplish experiments and simulations to evaluate satellite ACS [4]. Experimental test has the possibility of introducing more realism than the simulation; however, it has the difficulty of reproducing zero gravity and torque free space condition.

Examples of experimental platforms for investigating different aspects of the satellite dynamic and control system can be found in [5]. A classic case of a phenomenon not investigated experimentally before launch was the

\footnotetext{
*Correcponding author: L.C.G. Souza, National Institute for Space Research- INPE, Brazilian Aeronautical Company-Embraer, São Jose dos Campos, SP, Brasil. E-mail: gadelha@dem.inpe.br.
} 
dissipation energy effect that has altered the satellite Explorer I rotation [6]. An important aspect that must be first investigated through experimental procedure is the platform inertia parameters identification [7] and [8]. When inertia parameters are not well known the system can present some source of uncertainty [9]. An algorithm based on the least square method to identify mass parameters of a space vehicle in rotation during attitude maneuvers has been developed by [10], a methods with the same objectives, but based on Kaman filter theory also has been investigated by [11]. The H-infinity control technique was used by [12] to design robust control laws for a satellite composed of rigid and flexible panels. A good survey of the SDRE method can be found in [13] and its application to deal with non-liner plant [14], it is considered as the non-linear counterpart of LQR control. It linearizes the plant around the instantaneous point of operation and produces a constant state-space model of the system where a similar LQR control technique can be applied to design a specific controller. The process is repeated in the next sampling periods therefore producing and controlling several state dependent linear models out of a non-linear one. The SDRE method was applied by [15-17] for controlling a non-linear system similar to the six-degree of freedom satellite model considered in this paper. Kalman filter technique, when applied as state observer in conjunction with the SDRE method, allows the incorporation of non-linearities in the filter process. The uncertainties of the system [9] can be represented by process and measurements noise. In this paper the standard LQR linear controller and the SDRE controller associated with Kalman filter are applied to design a non linear controller for a non-linear plant of the DMC 3-D satellite simulator in the presence of noise. The performance and robustness of both methods are investigated by several simulations. Results have proven the reliability of SDRE method to design control algorithm to be implemented in an on board satellite computer.

\section{Simulator mathematical model}

Figure 1 shows the DMC 3-D simulator has a disk-shaped platform, supported on a plane with a spherical air bearing; details can be found in [6]. The platform can accommodate various satellites components; like sensors, actuators, computers and its respective interface and electronic. To represent the satellite model by the 3-D simulator one does not take into account the ground gravity gradient effects and that the air bearing creates torque free condition. As a result, basically, one follows the same step of modeling a rigid satellite with rotation in three axes free in space. The inertial reference system $F_{i}\left(I_{1}, I_{2}, I_{3}\right)$ is located in the centre of the spherical bearing. The orientation of the platform is given by the body reference system $\mathrm{F}_{\mathrm{b}}$ with respect to inertial system considering the principal axes of inertia. To describe the orientation of $F_{b}$, one uses Euler angles $\left(\theta_{1}, \theta_{2}, \theta_{3}\right)$ in the sequence 3-2-1, to guarantee that there is no singularity in the simulator attitude rotation.

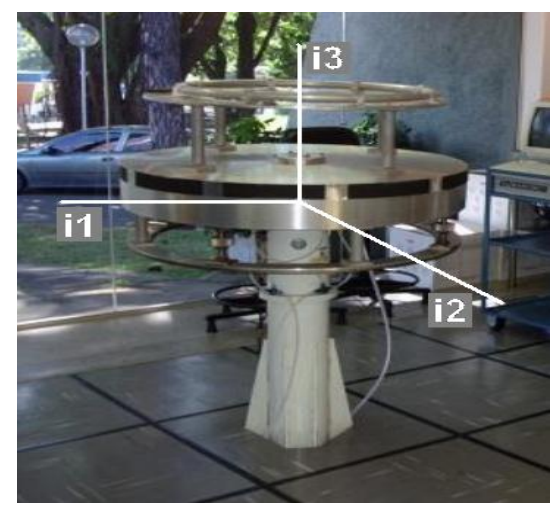

Fig. 1. The DMC satellite attitude control system simulator.

The equations of motions are obtained using Euler's angular moment theorem given by

$$
\dot{\vec{h}}=\vec{g}
$$


where $\vec{g}$ and $\vec{h}$ are the total torque and total angular moment of the system, which is given by

$$
\vec{h}=I \vec{\omega}+I_{w}(\vec{\Omega}+\vec{\omega})
$$

where $I=\operatorname{diag}\left(\mathrm{I}_{11}, \mathrm{I}_{22}, \mathrm{I}_{33}\right)$ is the system matrix inertia moment, $\vec{\omega}$ is the angular velocity of $F_{b}$, $\vec{I}_{w}=\operatorname{diag}\left(\mathrm{I}_{\mathrm{w} 1}, \mathrm{I}_{\mathrm{w} 2}, \mathrm{I}_{\mathrm{w} 3}\right)$ is the reaction wheel matrix inertia moment and $\Omega=\left(\Omega_{1}, \Omega_{2}, \Omega_{3}\right)$ are the reaction wheel angular velocity. Since $\vec{I}$ and $\vec{I}_{w}$ are constants in $F_{b}$, it is convenient to express (1) in this system.

Differentiating (1) and considering the external torque equal to zero, one has

$$
\dot{\vec{h}}+\vec{\omega}^{x} \vec{h}=0
$$

where $(.)^{\mathrm{x}}$ represents skew-symmetric matrix operator.

Substituting (2) into (3), the vector angular velocity of the platform is

$$
\dot{\vec{\omega}}=\left(I+I_{w}\right)^{-1}\left[-\vec{\omega}^{x}\left(I+I_{w}\right) \vec{\omega}-\vec{\omega}^{x} I_{w} \vec{\Omega}-I_{w} \dot{\vec{\Omega}}\right]
$$

The simulator attitude as function of the angular velocity is

$$
\left(\begin{array}{l}
\dot{\theta}_{1} \\
\dot{\theta}_{2} \\
\dot{\theta}_{3}
\end{array}\right)=\left(\begin{array}{ccc}
0 & \sin \theta_{3} / \cos \theta_{2} & \cos \theta_{3} / \cos \theta_{2} \\
0 & \cos \theta_{3} & -\sin \theta_{3} \\
1 & \sin \theta_{3} \sin \theta_{2} / \cos \theta_{2} & \cos \theta_{3} \sin \theta_{2} / \cos \theta_{2}
\end{array}\right)\left(\begin{array}{c}
\omega_{1} \\
\omega_{2} \\
\omega_{3}
\end{array}\right)
$$

\section{SDRE and Kalman filter methodologies}

Similar to the LQR technique, the SDRE method also has a performance criteria $J$ to be minimized given by

$$
J=\frac{1}{2} \int_{t_{0}}^{\infty} x^{T} Q(x) x+u^{T} R(x) u d t
$$

and the SDRE non-linear state space model is given by

$$
\begin{aligned}
& \dot{x}=f(x)+B(x) u \\
& s=C(x) x \\
& x(0)=x_{0}
\end{aligned}
$$

where $x(0)$ is the initial condition, $B \in \mathfrak{R}^{n x m}$ and $C \in \mathfrak{R}^{m x n}$ are the input and output matrices, which define the position and the type of actuator and sensor, $x \in \mathfrak{R}^{n}, u \in \mathfrak{R}^{m}$ e $s \in \mathfrak{R}^{s}$ are the states, the control and the output of the system, respectively. The stability condition requires that the weighting matrix $Q(x) \in \mathfrak{R}^{n x n}$ be positive semi-definite and $R(x) \in \mathfrak{R}^{m x m}$ be positive definite. These matrices are associated to penalizing the states and the control, respectively, and they work like tuning parameters in the control law performance.

The state variable feedback control law is given by

$$
u=-K(x) x
$$

with the state-dependent gain given by

$$
K(x)=-R^{-1} B^{T} P(x)
$$


and $P(x)$ is solution of the Riccati equation given by

$$
A^{T}(x) P(x)+P(x) A(x)-P(x) B R^{-1} B^{T} P(x)+Q=0
$$

Using the direct parameterization to transform the SDRE non nonlinear state model Eq. (7) into state dependent coefficient matrices of the state (SDC), one obtains system equations given by

$$
\dot{x}=A(x) x+B(x) u
$$

where $f(x)=A(x) x, A \in \mathfrak{R}^{n x n}$ is the state matrix, which in general is only unique if $\mathrm{x}$ is scalar. Depending of the non linear dynamics of the system, matrices $\mathrm{A}(\mathrm{x}), \mathrm{B}(\mathrm{x})$ and $\mathrm{C}(\mathrm{x})$ can be functions of the states or not.

Comparing with traditional non-linear control methods [18,19], the SDRE method has the advantage of avoiding intensive calculation, resulting in simpler control algorithms more appropriate to be implemented in satellite on-board computer. The SDRE linearizes the plant around the current operating point and creates constant state space matrices so that the LQR method can be used. This process is repeated in all steps, resulting in a point wise linear model from a non-linear model, so that the Riccati equation is solved and a control law is calculated also in each step.

Here the SDRE method is applied to design a satellite ACS simulator, where $\mathrm{A}(\mathrm{x})$ is the only non-linear state dependent matrix and the simulator model state vector $\mathrm{x}$ consists of the angle $\theta$ and angular velocity $\omega$ given by

$$
x=\left(\begin{array}{llllll}
\theta_{1} & \theta_{2} & \theta_{3} & \omega_{1} & \omega_{2} & \omega_{3}
\end{array}\right)^{T}
$$

After some manipulations, the non-linear state matrix A(x) and B given by Eqs (4) and (5) can be written in the state dependent coefficient (SDC) form by

$$
\begin{aligned}
& A(x)=\left(\begin{array}{cccccc}
0 & 0 & 0 & 0 & \frac{\sin \theta_{3}}{\cos \theta_{2}} & \frac{\cos \theta_{3}}{\cos \theta_{2}} \\
0 & 0 & 0 & 0 & \cos \theta_{3} & -\sin \theta_{3} \\
0 & 0 & 0 & 1 & \frac{\sin \theta_{3} \sin \theta_{2}}{\cos \theta_{2}} & \frac{\cos \theta_{3} \sin \theta_{2}}{\cos \theta_{2}} \\
0 & 0 & 0 & 0 & \frac{I_{22} \omega_{3}}{I_{11}+I_{w}} & \frac{-I_{33} \omega_{2}}{I_{11}+I_{w}} \\
0 & 0 & 0 & \frac{-I_{11} \omega_{3}}{I_{22}+I_{w}} & 0 & \frac{I_{33} \omega_{1}}{I_{22}+I_{w}} \\
0 & 0 & 0 & \frac{I_{11} \omega_{2}}{I_{33}+I_{w}} & \frac{-I_{22} \omega_{1}}{I_{33}+I_{w}} & 0 \\
0 & 0 & 0 \\
0 & 0 & 0 \\
0 & 0 & 0 \\
\frac{-I_{w}}{I_{11}+I_{w}} & 0 & 0 \\
0 & \frac{-I_{w}}{I_{22}+I_{w}} & 0 \\
0 & 0 & \frac{-I_{w}}{I_{33}+I_{w}}
\end{array}\right) \\
& 0 \\
& 0 \\
& 0
\end{aligned}
$$


One assumes that the $\mathrm{C}=\operatorname{diag}(1,1,1,1,1,1)$ and that the simulator uses torques actuators like reactions wheel and sensors of angle and angular velocities.

The SDRE theory presented up to here allows designing the control law without estimating the states; but for the case when some states are not available, one uses the Kalman filter, which also is adequate for on-board implementations because the estimation of the next state depends only on the previous and actual state, without keeping in the memory the history of all the states calculated. Besides, the Kalman filter takes into account the process noise w and the measures noise $\mathrm{z}$, which can be due to no modeled dynamics and/or external perturbation and due to sensor non linearities and/or noise, respectively. As a result, the system becomes

$$
\left.\begin{array}{l}
\dot{x}=A(x) x+B u+w \\
y=C x+z
\end{array}\right\}
$$

The Kalman filter algorithm consists basically of two steps; the time update and the measurement update, which is given by the following two sets of equations

$$
\left.\left.\begin{array}{l}
\hat{x}_{k}^{-}=A \hat{x}_{k-1}+B u_{k-1} \\
P_{k}^{-}=A P_{k-1} A^{T}+S_{w}
\end{array}\right\} \begin{array}{l}
K_{k}=P_{k}^{-} C^{T}\left(C P_{k}^{-} C^{T}+S_{z}\right)^{-1} \\
\hat{x}_{k}=\hat{x}_{k}^{-}+K_{k}\left(y_{k}-C \hat{x}_{k}^{-}\right) \\
P_{k}=\left(I-K_{k} C\right) P_{k}^{-}
\end{array}\right\}
$$

The matrix $P_{k}$ represents the covariance of the estimated state $\hat{x}$, and $K_{k}$ is the Kalman gain. One observes now that the matrix $A(x)$ is calculated in every step of estimating the states $\hat{x}, P_{k}$ and $K_{k}$.

\section{Simulations results}

One has used the matlab/simulink software to demonstrate the ability of the SDRE technique to control a non-linear plant in comparison with the LQR technique. The efficiency of the Kalman filter as a state estimator in the presence of noise is also shown. The controller performance and robustness requirements are: small overshoot and time of response and its ability to perform the control apart from the nonlinear terns of the plant, since one simulates big maneuvers in order to stress the non-linear terns of the plant. Considering that the simulator inertia parameters depend on the equipments distribution over it, one uses the following typical values $\mathrm{I}_{11}=\mathrm{I}_{22}=$ $1.17 \mathrm{Kg} \cdot \mathrm{m}^{2}$ and $\mathrm{I}_{33}=1.13 \mathrm{Kg} \cdot \mathrm{m}^{2}$; and for the wheel inertia $\mathrm{I}_{\mathrm{x}}=\mathrm{I}_{\mathrm{y}}=\mathrm{I}_{\mathrm{z}}=0.0018 \mathrm{Kg} \cdot \mathrm{m}^{2}$. One assumes typical mean values for the process noise $\mathrm{w}=0.0001 \mathrm{Nm}$ with covariance given by $\mathrm{S}_{\mathrm{w}}=10^{-8}$ and tipical mean values for sensor noise $\mathrm{z}$ in the angle $=0.2 \mathrm{deg}$ and in the angular velocity $=0.1 \mathrm{deg} / \mathrm{s}$ with covariance given by $\mathrm{S}_{\mathrm{z}}=\operatorname{diag}[0.04,0.01]$. Figure 2 shows the LQR and SDRE controllers without noise when the simulator initial attitude is zero and the final attitude is $(50,20,-30)$ deg which emphasizes the non-linear terms in the plant. One knows that the choice of weight matrix $\mathrm{Q}$ and $\mathrm{R}$ in the $\mathrm{LQR}$ problem is by tray and error. Therefore, after some simulations one gets $\mathrm{R}=$ $\operatorname{diag}(0.001,0.001,0.001)$ and $\mathrm{Q}=\operatorname{diag}(1,1,1,100,100,100)$ to the SDRE controller manager to have better performance than $\mathrm{LQR}$ controller using the same matrix $\mathrm{R}$ and $\mathrm{Q}$. It is important to stress that this performance is a function of the weighting matrices of the LQR and SDRE controllers.

Figure 3 shows SDRE controllers without noise for the previous maneuver; however, only the angles are fed back. Without angular velocity sensor, the SDRE controller performance is degraded showing the need for Kalman filter to estimates the states that are not available.

Figure 4 shows the SDRE controllers in the presence of noise for the same large attitude maneuvers as before. It is observed that the SDRE controller performance is quite similar when there is no noise. This results show that the SDRE controller has the same robust properties of the LQR controller when all the states are available for feedback. 

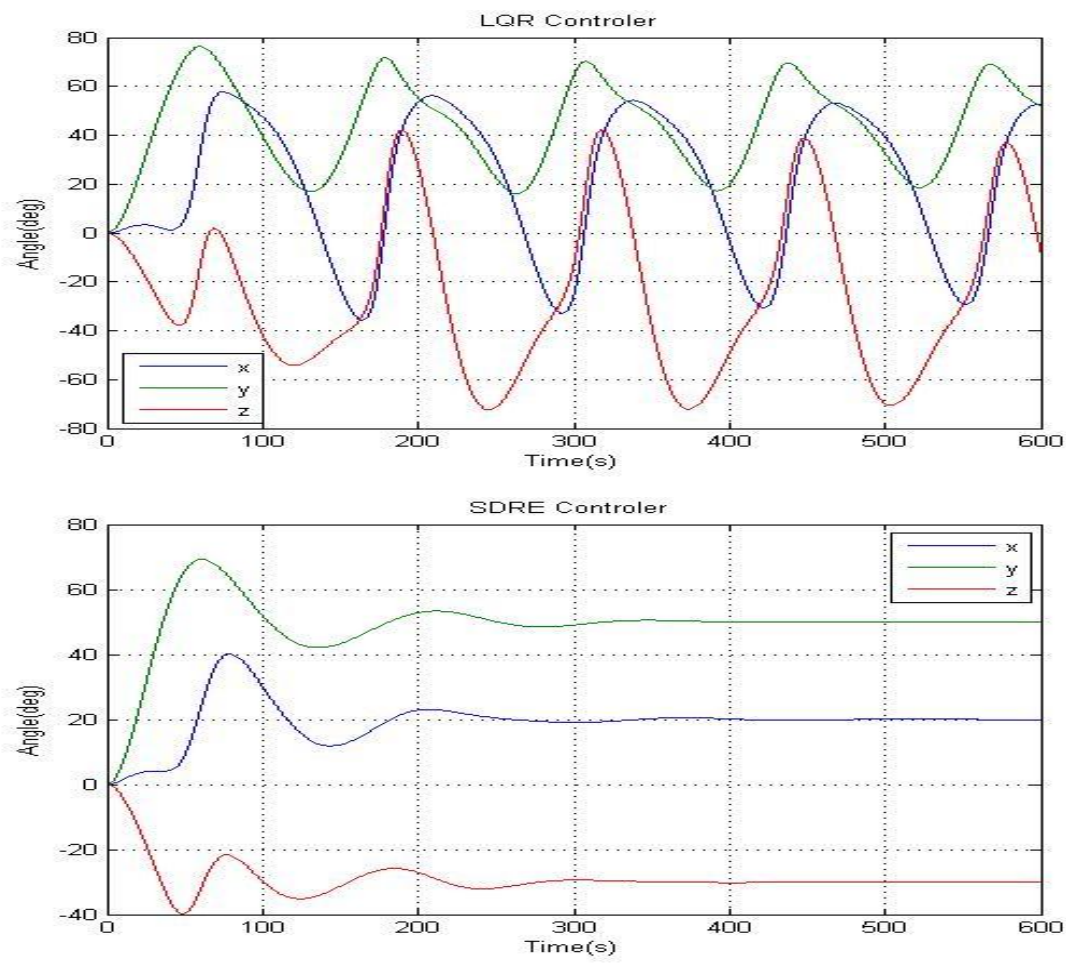

Fig. 2. The LQR and SDRE controllers for large maneuvers.

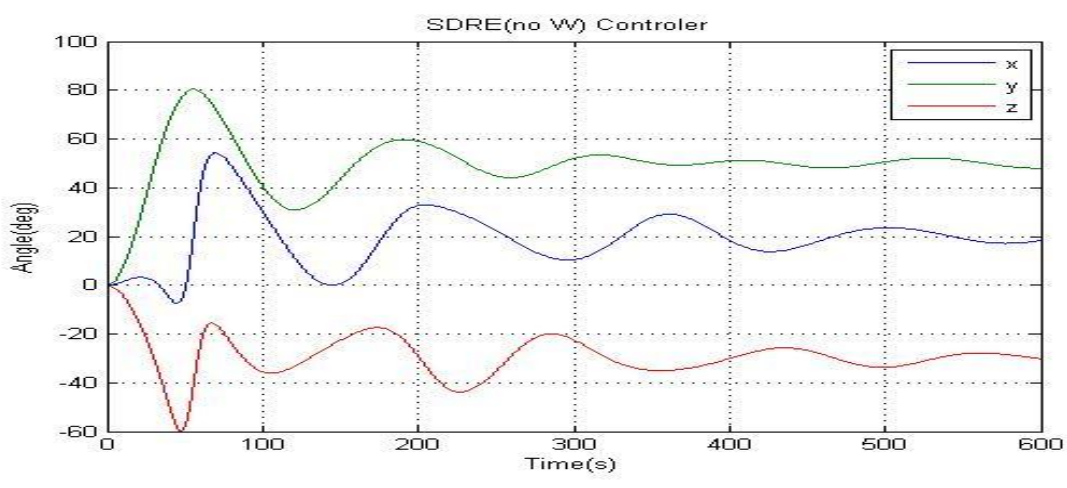

Fig. 3. The SDRE controllers with only angle to feedback

One should keep in mind that noise effect is very important when the controllers pointing precision is very demanding.

Figure 5 shows the SDRE controllers in the presence of noise for the same large attitude maneuvers, having only the angular measurements for feedback. It is possible to observe that the SDRE controller performance has been degraded due to the noise presence and the unavailability of all the states. In this case the introduction of the Kalman filter in the control system to estimates all the states is fundamental.

Figure 6 shows the angles maneuver of the SDRE controllers in the presence of noise for the same large attitude maneuvers as before. However, now a Kalman filter is used to estimate angle and angular velocities, using a sampling time of $0.1 \mathrm{~s}$. One observes that the SDRE controller performance has been improved showing that better measurements for feedback are very important when large maneuvers end in stringent pointing accuracy. 


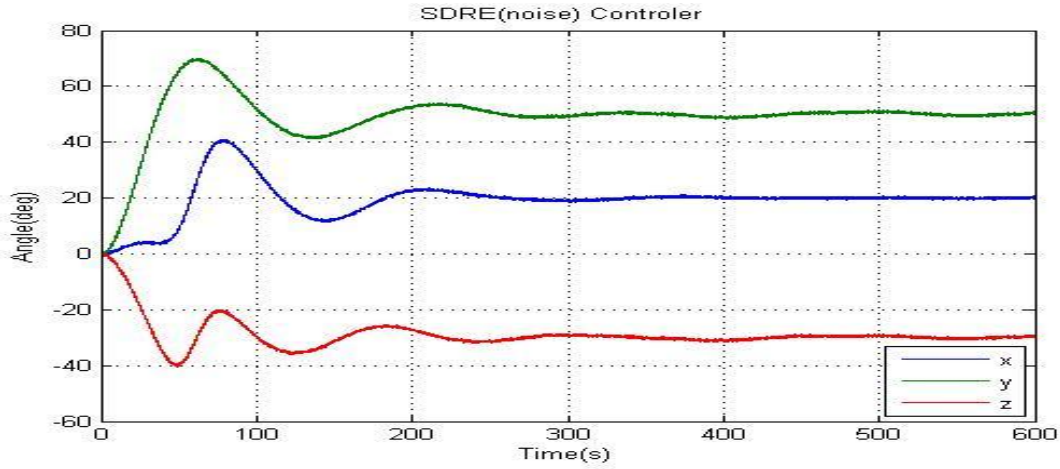

Fig. 4. The SDRE controllers with system noise.

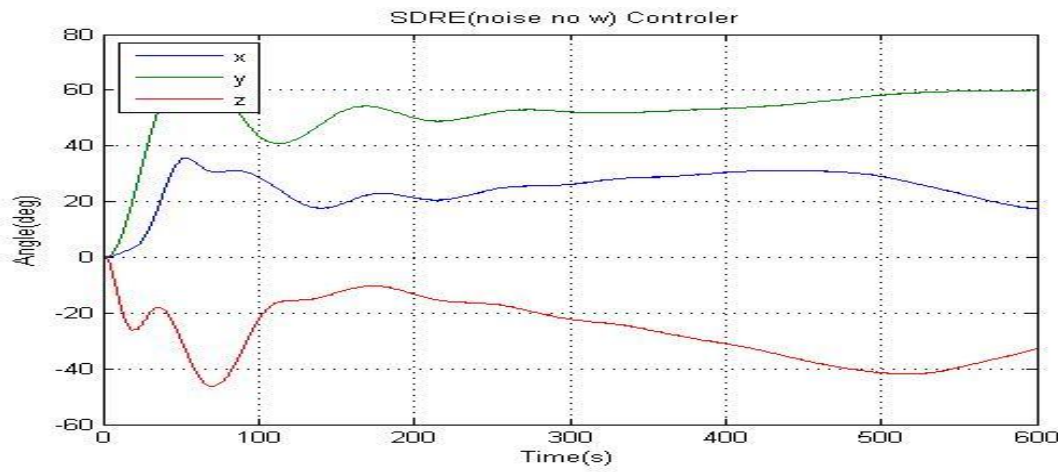

Fig. 5. The SDRE controllers with noise and only angle to feedback.

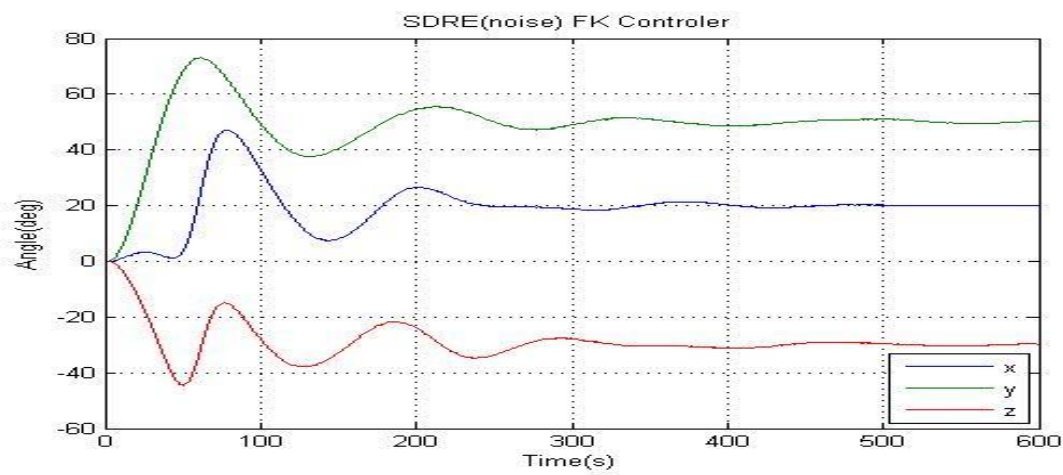

Fig. 6. The SDRE controllers with noise and Kalman filter.

\section{Summary}

This paper presents the DMC 3-D satellite simulator mathematical model and the design of its ACS based on the SDRE method associated with Kalman filter technique. The satellite simulator model is generic since its equation of motion depends only on its inertia parameters. Simulation has shown that the SDRE controller has superior performance than the LQR controller for large angle maneuvers when the plant non-linear terns are relevant. The LQR controller performance reaches instability where the final attitude angle is far from the origin. On the other hand, the 
SDRE controller is able to maintain the same level of performance in any region, demonstrating its ability to control non-linear plants. Regarding the presence of noise and Kalman filter implementation together with SDRE technique, one observes that the noise levels affect the SDRE controller performance when the pointing accuracy is stringent, being less relevant when the simulator performs large angle maneuvers. In general, one observes that the SDRE method keeps the same robust properties of the LQR; however, losing performance when there is no availability of all states for feedback. Finally, the simulations results have proven the reliability of SDRE method to design control algorithm to be implemented in an on board satellite computer, since its gains are constant similar to the LQR.

\section{References}

[1] L.C.G. Souza, Design of satellite control system using optimal non-linear theory, Mechanics Based Design of Structures and Machine 1 (2008), 1-20.

[2] B.N. Agrawal and R.E. Rasmussen, Air Bearing Based Satellite Attitude Dynamics Simulator for Control Software Research and Development, in: Technologies for Synthetic Environments: Hardware-in-the-Loop Testing VI, Robert Lee Murrer, Jr., ed., Proceedings of SPIE Vol. 4366, 2001, pp. 204-214.

[3] R.H. Cannon and D.E. Rosenthal, Experiments in control of flexible structures with noncolocated sensors and actuators, Journal of Guidance, Control and Dynamics 7(5) (1984), 546-553.

[4] C.D. Hall, Tsiotras and H. Shen, Tracking rigid body motion using thrusters and momentum wheels, Journal of the Astronautical Sciences 3 (2002), 13-20.

[5] G.T. Conti and L.C.G. Souza, Satellite attitude system simulator, Journal of Sound and Vibration 15 (2008), 392-395.

[6] M.H. Kaplan, Modern Spacecraft Dynamics and Control, 1976, John Wiley \& Sons, Inc., USA.

[7] A.Y. Lee and J.A. Wertz, In-flight estimation of the Cassini Spacecraft inertia tensor, Journal Spacecraft and Rokect 39(1) (2002), $153-155$.

[8] S. Tanygin and T. Williams, Mass property estimation using coasting maneuvers, Journal of Guidance, Control, and Dynamics 11 (May-June, 1997), 625-632.

[9] L.C.G. Souza, Robust control design for a flexible space system with mixed uncertain model, Journal of the Brazilian Society of Mechanical Sciences and Engineering XVI (1994), 116-123.

[10] L.C.G. Souza, Inertia parameters estimation of a satellite simulator, Advances in Astronautical Sciences 133 (2008), $969-979$.

[11] J. Ahmed, V.T. Coppola and D. e Bernstein, Adaptative asymptotic tracking of spacecraft attitude motion with inertia matrix identification, Journal of Guidance, Control, and Dynamics 21 (1998), 684-691.

[12] X.C.M. Cubillos and L.C.G. Souza, Using H-infinity control method to design an attitude control system of rigid-flexible satellite, Mathematical Problem Engineering 1 (2009), 1-10.

[13] T. Çimen, State-Dependent Riccati Equation (SDRE) Control: A Survey, Proceedings of the 17th World Congress - The International Federation of Automatic Control, Seoul, Korea, July 6-11, 2008.

[14] G.R. Guidoni and L.C.G. Souza, Application of the SDRE method to design an attitude control system simulator, Advances in Astronautical Sciences 134 (2009), 2251-2258.

[15] T.D. Donald and R.J. Coultier, Position and Attitude control of a Spacecrafts Using the State-Dependent Riccati Equation Technique, Proceedings of the American Control Conference, Chicago, USA, 2000.

[16] P.K. Menon, Real Time Computational Methods for SDRE Non-linear Control of Missiles, Proceedings of the American Control Conference, Anchorage, AK, USA, May 8-10, 2002.

[17] E.V. Bergmann and J. e Dzielski, Spacecraft mass property identification with torque-generating control, Journal of Guidance, Control and Dynamics 13 (Jan-Feb, 1990), 99-103. .

[18] I. Mainenti-Lopes, L.C.G. Souza, F.L. Sousa and A.P.C. Cuco, Multi-objective Generalized Extremal Optimization with real codification and its application in satellite attitude control, Proceedings of 19th International Congress of Mechanical Engineering - COBEM, Gramado, Brasil, 2009.

[19] A. Isidori, Nonlinear Control System, Spring -Verlag, London, ISBN: 3-540-199-16-0, 1995. 

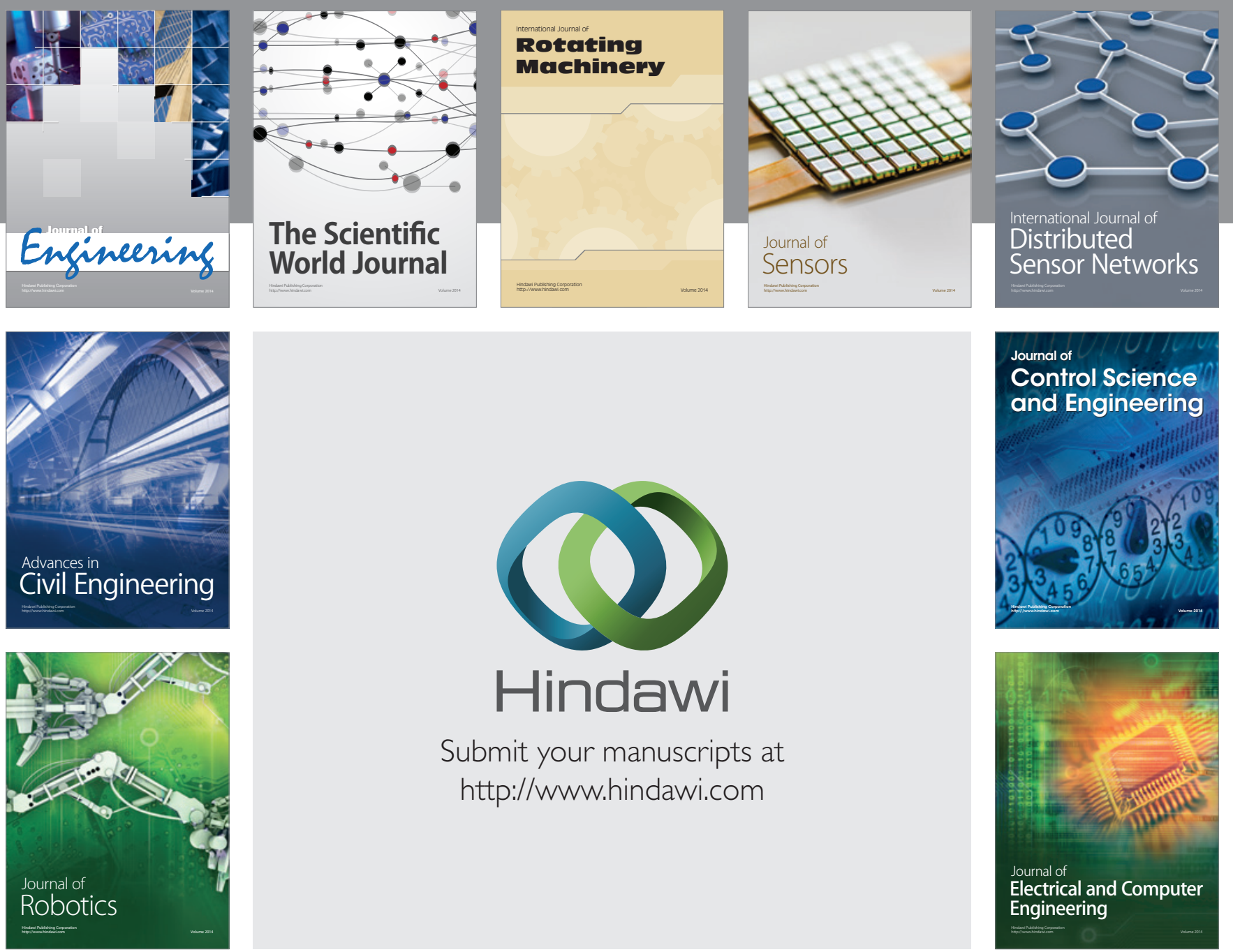

Submit your manuscripts at

http://www.hindawi.com
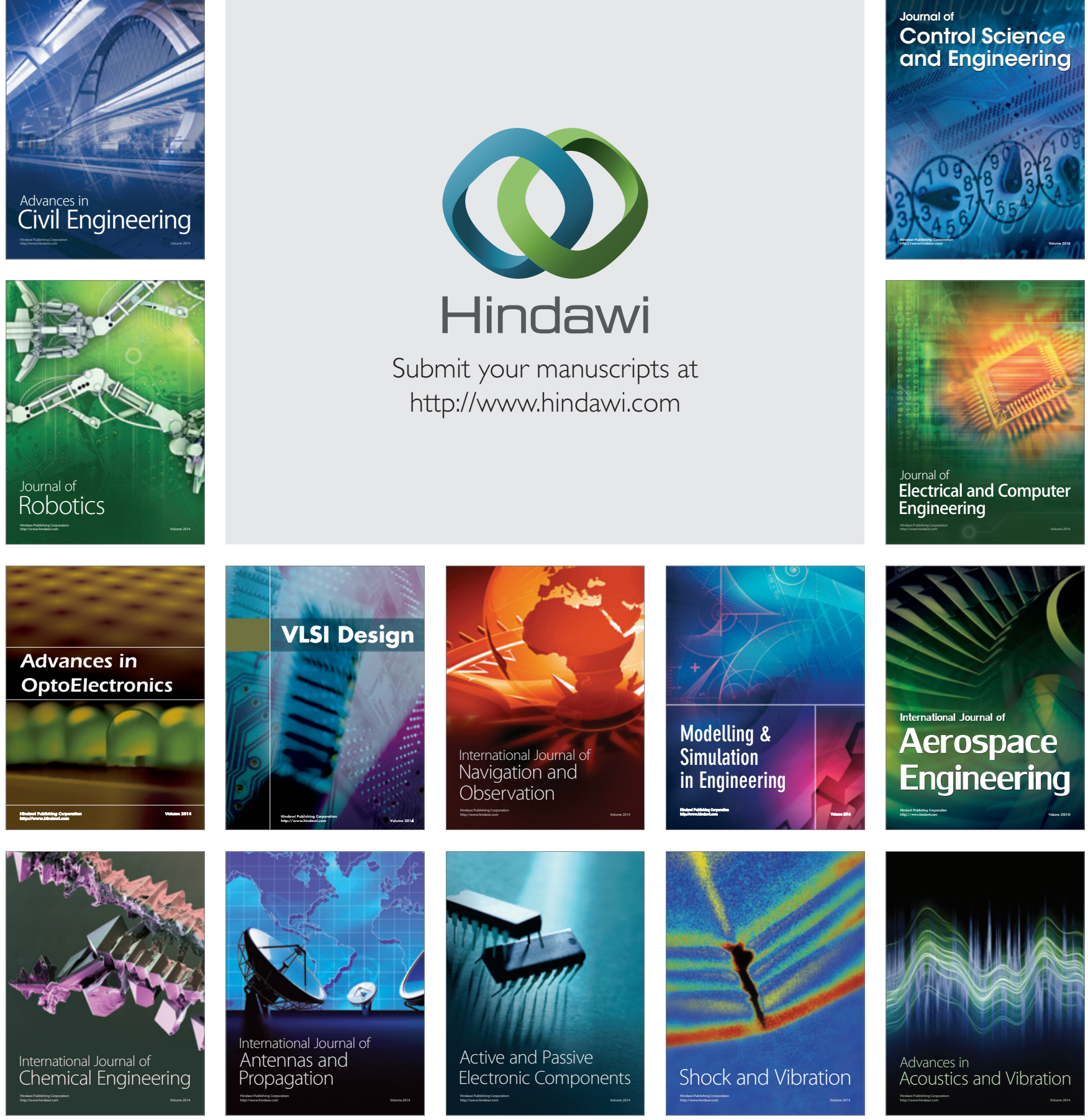